\title{
-Technology Report-
}

\section{Assisted Hatching of Poor-quality Bovine Embryos Increases Pregnancy Success Rate After Embryo Transfer}

\author{
Atsushi TANIYAMA ${ }^{1,3)}$, Yasutaka WATANABE ${ }^{2)}$, Youji NISHINO2), Tetsurou INOUE1), \\ Yasuho TAURA $^{3)}$, Mitsuhiro TAKAGII), Chikara KUBOTA ${ }^{3)}$ and Takeshige OTOI ${ }^{3)}$ \\ ${ }^{1)}$ Nagasaki Prefectural Livestock Experiment Station, Nagasaki 859-1404, ${ }^{2)}$ Nankou ET Center, Nagasaki $859-1404$ and \\ ${ }^{3)}$ The United Graduate School of Veterinary Science, Yamaguchi University, Yamaguchi 753-8515, Japan
}

\begin{abstract}
Embryos of good, fair and poor quality were collected from superovulated cows and subjected to zona cutting (ZC) treatment using a needle under either an inverted microscope or a stereomicroscope. One (single transfer) or 2 (twin transfer) embryos with or without prior ZC treatment were transferred nonsurgically to recipients. Without the ZC treatment, lower embryonic quality resulted in lower pregnancy success rates. However, the ZC treatment increased the pregnancy success rate following transfer of poor-quality embryos, but not the pregnancy rate after transfer of good- or fair-quality embryos. No differences were observed between the pregnancy success rates after the transfer of embryos treated under the inverted microscope and those after transfer of embryos treated under the stereomicroscope, and this was the same after single and twin transfer. Moreover, ZC treatment of embryos prior to transfer did not result in an increased abortion rate, irrespective of the number of transferred embryos. In conclusion, $\mathrm{ZC}$ treatment improves pregnancy success rates following transfer of poor-quality embryos. Moreover, the results indicate that ZC treatment by using a stereomicroscope is practical for on-farm application.
\end{abstract}

Key words: Assisted hatching, Micromanipulation, Pregnancy, Zona cutting

(J. Reprod. Dev. 57: 543-546, 2011)

$\mathbf{E}$ mbryo transfer is a commonly used method in animal reproduction and, more specifically, in genetic improvement of cattle. The quality of donor embryos tends to vary greatly, and a strong relation has been described between the embryonic quality and pregnancy success rate $[1,2]$. Poor-quality embryos show low viability after cryopreservation, and their transfer results in low pregnancy success rates. Therefore, poor-quality embryos are frequently discarded, even if the recipients are available at the day of collection. This results in considerable economic loss for the farmers concerned. Therefore, it is important to improve the survival of poor-quality embryos after embryo transfer.

It has been known that the hatching of the blastocyst from the zona pellucida is a key event in embryo development. Embryo transfer cannot result in a successful pregnancy if the blastocyst does not hatch within a limited time after implantation into the uterus. It has been demonstrated that zona slitting in human embryos increases the rate of hatching significantly [3]. In addition, Check et al. [4] reported that assisted hatching using the zonadrilling technique is not detrimental for frozen-thawed human embryos and that it is beneficial for embryonic development upon transfer. However, although embryo transfer is well established in cattle, little information is available on assisted hatching techniques for poor-quality bovine embryos. Moreover, the micromanipulation techniques involved in zona-drilling and zona-cutting (ZC) require large and expensive laboratory equipment, including an

Received: June 23, 2010

Accepted: April 18, 2011

Published online in J-STAGE: May 23, 2011

(C)2011 by the Society for Reproduction and Development

Correspondence: T Otoi (e-mail: otoi@yamaguchi-u.ac.jp) inverted microscope with a micromanipulator. For this reason, these techniques are not practical for use on farms and cannot form the basis of an acceptable on-farm procedure. The use of a stereomicroscope for $\mathrm{ZC}$ could overcome this problem; the stereomicroscope is a smaller microscope and can easily be carried, even with a micromanipulator attached to it. Moreover, the use of a stereomicroscope reduces the costs associated with micromanipulation.

It has been shown previously that zona-slitting using a microsurgical blade improved the development of fresh embryos with poorquality, but not the pregnancy rates after transfer [5]. It appears that mechanical zona dissection using a microsurgical blade increases the risk of embryo damage. This is probably due to the thickness of the blade, which ranges from one-quarter to one-third of the embryonic diameter. Alternatively, a needle can be applied to puncture the zona pellucida without causing damage to the cell, enabling easy and stable manipulation while dissecting the zona pellucida of the embryo [6].

The objective of this study was to examine whether ZC of poorquality embryos with a needle can improve pregnancy success rates following transfer. Moreover, we investigated whether our setup for zona cutting of embryos, consisting of a micromanipulator mounted on a stereomicroscope, is practical and acceptable for onfarm use.

We examined the effects of embryo quality and ZC treatment on the pregnancy success rates following single embryo transfer (Table 1). The pregnancy success rate after transfer of poor-quality embryos without $\mathrm{ZC}$ treatment was significantly lower than that following good-quality embryo transfer $(\mathrm{P}<0.05)$. In contrast, when the embryos received $\mathrm{ZC}$ treatment prior to embryo transfer, the differences in the pregnancy success rates related to the 3 
Table 1. Effects of embryo quality and zona cutting (ZC) on pregnancy success rates following single embryo transfer

\begin{tabular}{|c|c|c|}
\hline \multirow[t]{2}{*}{ Embryo quality } & \multicolumn{2}{|c|}{ No. of pregnancies $(\%)^{*}$} \\
\hline & Without ZC & With ZC \\
\hline Good & $18 / 23(78.3)^{\mathrm{a}}$ & (75.0) \\
\hline Fair & $7 / 16 \quad(43.8)^{\mathrm{b}}$ & $19 / 42 \quad(45.2)$ \\
\hline Poor & $6 / 26 \quad(23.1)^{\mathrm{b}, \mathrm{A}}$ & $48 / 108(44.4)^{\mathrm{B}}$ \\
\hline
\end{tabular}

* Number of pregnant cows/number of cows with embryos transferred. ${ }^{a-b}$ Values with different superscripts in the same column differ significantly $(\mathrm{P}<0.05)$. ${ }^{\mathrm{A}-\mathrm{B}}$ Values with different superscripts in the same row differ significantly $(\mathrm{P}<0.05)$.

groups of embryos were no longer significant. However, the fairand poor-quality embryos still fared worse compared with the good-quality embryos $(\mathrm{P}<0.1)$. The pregnancy success rate following transfer of poor-quality embryos that received $\mathrm{ZC}$ treatment was significantly higher $(\mathrm{P}<0.05)$ than that following transfer of poor-quality embryos without $\mathrm{ZC}$ treatment.

We examined the effects of $\mathrm{ZC}$ treatment and of the number of transferred embryos on the pregnancy success rates after transfer of poor-quality embryos (Table 2). For singly-transferred, poor-quality embryos, the pregnancy success rate after transfer of ZC-treated embryos tended to be higher than that after transfer of untreated embryos, irrespective of the microscope used. No apparent effect of ZC treatment was observed on the pregnancy success rates after twin embryo transfer. However, if the poor-quality embryos received $\mathrm{ZC}$ treatment under an inverted microscope, the pregnancy success rate was significantly higher after twin transfer than after single transfer.

The data for 2 different microscopes were combined to assess the effects of ZC treatment and of the number of transferred embryos on the number of calves produced following transfer of poor-quality embryos. A total of 77 pregnancies were pursued until parturition. No differences in abortion rates of embryos without the ZC treatment were found between the single embryo transfer group $(0 \%, 0 / 5)$ and twin embryo transfer group $(33.3 \%, 2 /$ 6). Similarly, the ZC treatment did not make any difference in the abortion rates of embryos between the single transfer $(16.1 \%, 5 / 31)$ and twin transfer $(28.6 \%, 10 / 35)$ groups. In the twin embryo transfer group, however, the abortion rates of embryos with and without the $\mathrm{ZC}$ treatment reached $28 \%$ and $33 \%$, respectively. In the case of twin transfer, the $\mathrm{ZC}$ treatment resulted in an increase in the number of twin births after parturition compared with single transfer $(48.0 \%, 12 / 25$, vs. $3.8 \%, 1 / 26 ; \mathrm{P}<0.01)$.

The quality of fresh and frozen-thawed embryos has been suggested to be the determining factor in the pregnancy success rates after embryo transfer [1]. Lindner and Wright [2] reported that pregnancy success rates following transfer of embryos can be predicted based on the quality of bovine embryos, as determined using morphological criteria. They demonstrated that embryos that were classified as excellent, good, fair and poor yielded pregnancy success rates of 45, 44, 27 and 20\%, respectively. Embryos classified as "excellent" and "good" yielded the highest pregnancy rates,
Table 2. Effects of zona cutting (ZC) and number of transferred embryos on pregnancy rates following poor-quality embryo transfer

\begin{tabular}{|c|c|c|}
\hline \multirow[t]{2}{*}{ ZC method } & \multicolumn{2}{|c|}{ No. of pregnancies $(\%)^{*}$} \\
\hline & Single embryo transfer & Twin embryo transfer \\
\hline Control** & $(23.1)^{\mathrm{a}}$ & $6 / 14 \quad(42.9)$ \\
\hline Inverted microscope & $24 / 56(42.9)^{\mathrm{a}, \mathrm{b}, \mathrm{A}}$ & $23 / 35(65.7)^{\mathrm{B}}$ \\
\hline Stereomicroscope & $24 / 52(46.2)^{\mathrm{b}}$ & $44 / 77 \quad(57.1)$ \\
\hline
\end{tabular}

* Number of pregnant cows/number of cows with embryos transferred. ** Embryos were transferred into recipients without prior ZC treatment.

${ }^{\mathrm{a}-\mathrm{b}}$ Values with different superscripts in the same column differ significantly $(\mathrm{P}<0.05)$. ${ }^{\mathrm{A}-\mathrm{B}}$ Values with different superscripts in the same row differ significantly $(\mathrm{P}<0.05)$.

whereas poor-quality embryos resulted in the lowest success rate. In agreement with this, we observed lower pregnancy success rates for embryos that scored lower on these morphological criteria. Even with embryos that had been subjected to $\mathrm{ZC}$ treatment prior to embryo transfer, the pregnancy success rates depended on embryonic quality. Among the embryos collected from 273 superovulated donor cows, the poor-quality embryos represented approximately $19 \%$ of all transferable embryos (data not shown). Considering that these embryos are suitable for neither transfer nor freezing, the development of strategies to improve the viability of poor-quality embryos is of fundamental value for the feasibility of embryo transfer programs.

Our results showed that ZC treatment of poor-quality embryos prior to transfer using a micromanipulator equipped with a needle increases the pregnancy success rate. However, the same treatment of good-quality and fair-quality embryos did not affect the pregnancy success rates upon transfer of these embryos. These results are in agreement with those reported by Lopatarova et al. [5]. They reported that the zona-slit treatment using a microsurgical blade improved embryo development to the hatching and hatched blastocyst stages of grade 2-3 embryos, but not of grade 1 embryos. In addition, they demonstrated that the zona-slit treatment did not negatively affect the implantation rates after embryo transfer. Taking previous results together with our findings, it can be concluded that the ZC treatment using a needle does not harm the embryos and that it enhances the pregnancy success rates after the transfer of poor-quality embryos.

One method that is applied to increase pregnancy success rates in recipients of bovine embryos is the transfer of 2 embryos into the uterine horn ipsilateral to the corpus luteum. The transfer of 2 embryos into the ipsilateral uterine horn likely increases the amounts of interferon- $\tau$ and other embryonic signaling molecules in the uterus needed to maintain pregnancy and prevent luteolysis $[8,9]$. Moreover, it has been suggested that cotransfer of embryonic vesicles increases trophoblastic signals and results in higher pregnancy rates in embryo transfer recipients [10]. In the present study, all poor-quality embryos, both with and without ZC treatment, were transferred into the ipsilateral uterine horn. With regard to the poor-quality embryos, twin embryo transfer of embryos that had undergone ZC treatment using the inverted microscope resulted in the highest pregnancy success rate (Table 2). No statis- 


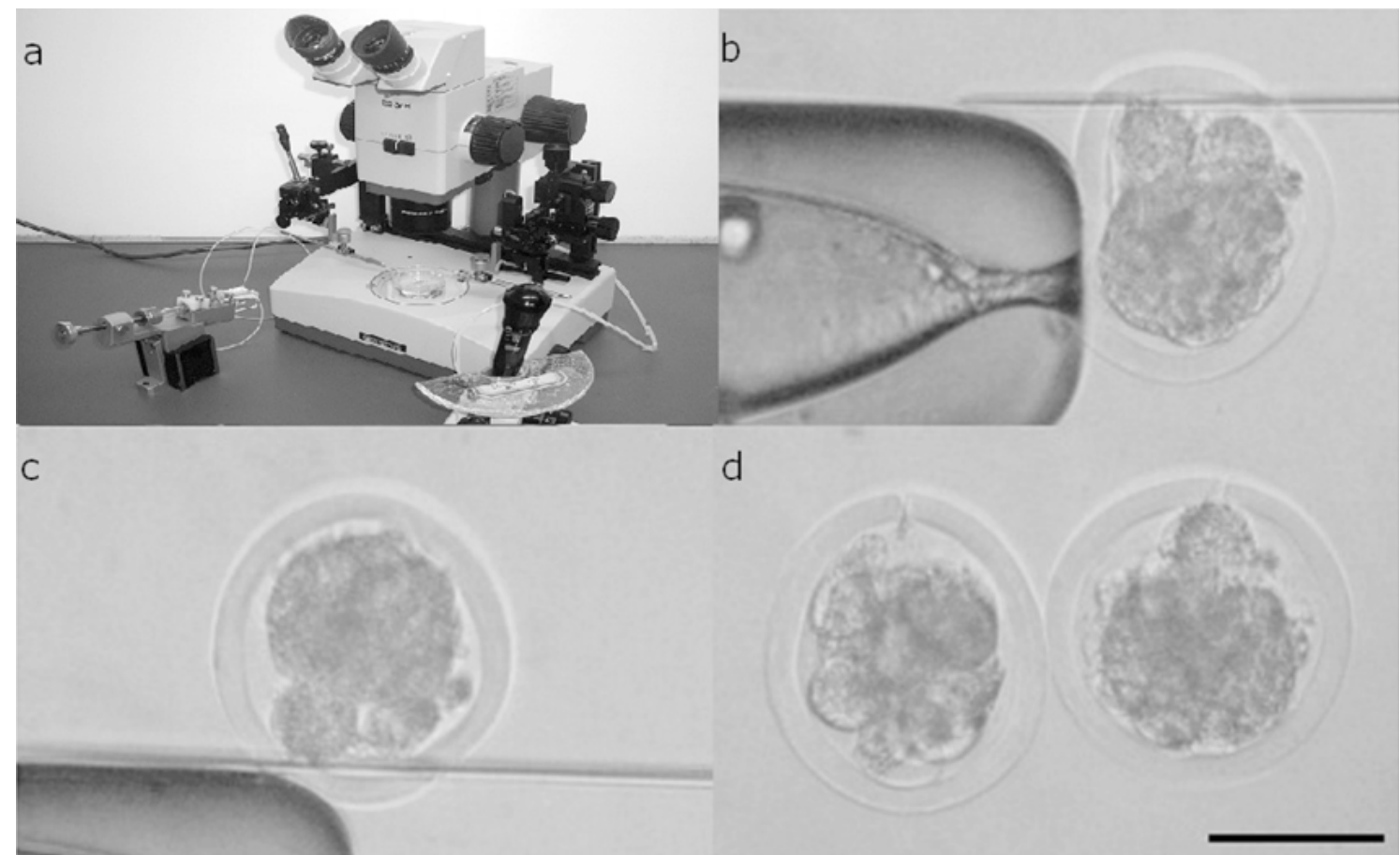

Fig. 1. a: Setup for micromanipulation: a Narishige micromanipulator (MMO-202ND; Narishige) used for the zona cutting of embryos was mounted on a stereomicroscope (SZH10-ILLK; Olympus) and directed with a three-axis, hydraulic joystick (MO-202; Narishige). A two-axis joystick (MN-151; Narishige) was coupled to the stage micromanipulator. b: The cutting needle was introduced through the zona. c: The holding pipette and cutting needle were rubbed on the trapped area until the embryo fell from the needle. d: Embryos with a slit in the zona pellucida. The scale bar represents $100 \mu \mathrm{m}$.

tically significant differences were found between the pregnancy success rates from the other groups, although twin transfer resulted in slightly higher rates than single embryo transfer.

$\mathrm{ZC}$ treatment had a positive effect on the pregnancy success rate after single embryo transfer but not on that after twin embryo transfer. Numabe et al. [11] reported that the pregnancy success rate after twin embryo transfer of in vitro-produced bovine embryos was higher than that resulting from single embryo transfer. However, no such effect was observed for in vivo-developed embryos. In their view, these differences in pregnancy rates reflect the differences in quality of in vivo and in vitro embryos. Therefore, pregnancy success rates after transfer of poor-quality embryos may be improved by twin transfer or, alternatively, by ZC treatment when only a single embryo is transferred. On the other hand, twin embryo transfer after ZC treatment increased the birth rates of twin calves. Moreover, the $\mathrm{ZC}$ treatment did not increase the abortion rate in recipients, irrespective of the number of transferred embryos. Although the numbers recorded in this study are insufficient to discern differences with statistical significance, the results suggest that the combination of ZC treatment and twin embryo transfer in poor-quality embryos may be optimal for calf production. Moreover, no differences were observed between the pregnancy success rates after the transfer of embryos treated under the inverted microscope and those after transfer of embryos treated under the stereomicroscope, and this was the same after single and twin transfer. In the farm field where embryos were collected, we had used a stereomicroscope equipped with a micromanipulator. This indicates that micromanipulation using a stereomicroscope is a practical and acceptable alternative for on-farm use.

In conclusion, based on the outcome of this study, the $\mathrm{ZC}$ treatment using a needle is not harmful for the viability of embryos after embryo transfer. The pregnancy success rates and calf production after transfer of poor-quality embryos can be improved by the combination of ZC treatment and twin embryo transfer. Moreover, the use of a stereomicroscope equipped with a needle proved practical and acceptable for on-farm use.

\section{Methods}

\section{Embryo production}

A field trial was set up in cooperation with the Nankou Embryo Transfer Center (Nagasaki, Japan). All embryos were recovered from Japanese Black donors (2-15 years old), which were bred at the Nagasaki Prefectural Livestock Experiment Station or farm, by the same veterinarian. Superovulation was induced by intramuscular administration of $14 \mathrm{AU}$ follicle stimulating hormone (FSH, Antrin; Kawasaki Seiyaku, Kawasaki, Japan) administered in a series of decreasing doses over a 4-day period beginning from day 9 of the estrous cycle. Each donor was given 2 doses $(20 \mathrm{mg} / \mathrm{dose})$ of prostaglandin $\mathrm{F}_{2}$ alpha (Pronalgon F; Takeda Pharmaceutical, Osaka, Japan) along with the fifth and sixth FSH treatments. Donors were inseminated with frozen-thawed semen from Japa- 
nese Black bulls at 12 and $24 \mathrm{~h}$ after detection of estrus. Embryos were recovered nonsurgically using a balloon catheter 7 days after the first insemination (day 0). After collection, the embryos were classified according to morphological observations.

\section{Assessment of embryo quality}

The embryos were examined for their morphology under a stereomicroscope and classified according to the criteria defined by the International Embryo Transfer Society [7]. The quality grade was assessed as good (code 1), fair (between codes 1 and 2), poor (code 2 ) or degenerated. Embryos were classed as "good" if their appearance was as expected for the respective stages of development, with few, if any extraneous, degenerated cells. Fair-quality embryos had small imperfections, and up to $20 \%$ of the cells could be degenerated. In poor-quality embryos, more than $30 \%$ of the cells were degenerated, but they still possessed a mass of cells that appeared viable.

\section{Embryo micromanipulation and embryo transfer}

Assisted hatching of embryos was performed by making a slit in the zona pellucida using a micromanipulator (MMO-202ND; Narishige, Tokyo, Japan) equipped with a needle (\#3-000-210-G; Drummond Scientific, PA, USA) and a pipette (G-1; Narishige) attached to an inverted microscope (IMT-2-12; Olympus, Tokyo, Japan) or stereomicroscope (SZH10-ILLK; Olympus; Fig. 1a). The inverted microscope and stereomicroscope were used in the laboratory and in the field facility where embryos were collected. Embryos were placed on a dish in a microdrop of Dulbecco's phosphate-buffered saline (PBS; Invitrogen, Carlsbad, CA, USA) containing $20 \%$ calf serum (Invitrogen) and overlaid with mineral oil. Each embryo was secured in position with a holding pipette and rotated as required to form a slit in the zona pellucida. The needle was introduced through the zona in such a way that its tip entered the perivitelline space and pierced the zona pellucida in 2 positions (coming in and going out). An area of the zona was thereby trapped between the 2 points that had been pierced by the needle (Fig. 1b). This area encompassed about $20 \%$ of the embryo's circumference. The suction in the holding pipette was then released so that the embryo remained suspended on the needle. The holding pipette and needle were rubbed against each other in order to slice the trapped area (Fig. 1c). This resulted in a slit in the zona pellucida (Fig. 1d). ZC of embryos was usually completed within $1 \mathrm{~min}$, even when the procedure was carried out under the stereomicroscope under farm conditions.

The embryos were transferred to 353 Holstein recipients (16 heifers and 337 cows) in a randomized fashion. Good- and fairquality embryos (with and without $\mathrm{ZC}$ treatment) were singly transferred to 35 and 58 recipients, respectively. One (single transfer) and 2 (twin transfer) poor-quality embryos (with or without the ZC treatment) were transferred to 134 and 126 recipients, respectively. The embryos were transferred nonsurgically into the uterine horn ipsilateral to the ovary bearing the corpus luteum on day 7 of the estrous cycle. Generally, conception rates after embryo transfer are known to be affected by the condition of the recipients and the skill with which the techniques are performed. For this study, 4 technicians performed all the transfers, and the same standards were used throughout the study to select recipient cows. Pregnancy was determined by rectum palpation on days $45-60$ after estrus.

\section{Statistical analysis}

The rates of pregnancy, abortion and twin birth after embryo transfer were analyzed using chi-square analysis with Yates' correction. When expected values were $\leq 5$, Fisher's exact probability test was used. Statistical significance was inferred for $\mathrm{P}<0.05$, while tendencies were reported at $\mathrm{P}<0.1$.

\section{References}

1. Hasler JF. Factors affecting frozen and fresh embryo transfer pregnancy rates in cattle Theriogenology 2001; 56: 1401-1415.

2. Lindner GM, Wright RW. Bovine embryo morphology and evaluation. Theriogenology 1983; 20: 407-416.

3. Dokras A, Ross C, Gosden B, Sargent IL, Barlow DH. Micromanipulation of human embryos to assist hatching. Fertil Steril 1994; 61: 514-520.

4. Check JH, Hoover L, Nazari A, O'Shaughnessy A, Summers D. The effect of assisted hatching on pregnancy rates after frozen embryo transfer. Fertil Steril 1996; 65: 254 257

5. Lopatarova M, Holy L, Vinkler A. Effect on survival of micromanipulating the zona pellucida of bovine embryos. Acta Vet Brno 2001; 70: 49-56.

6. Lyu QF, Wu LQ, Li YP, Pan Q, Liu DE, Xia K, Liang DS, Cai F, Long ZG, Dai HP, Xia JH. An improved mechanical technique for assisted hatching. Hum Reprod 2005; 20 1619-1623.

7. Robertson I, Nelson RE. Certification and identification of the embryo. In: Stringfellow DA, Seidel SM (eds.), Manual of the Intermational Embryo Transfer Society, 3rd ed, 1998: 103-134.

8. Peterson AJ, Lee RS. Improving successful pregnancies after embryo transfer. Theriogenology 2003; 59: 687-697.

9. Kubisch HM, Sirisathien S, Bosch P, Hernandez-Fonseca HJ, Clements G, Liukkonen JR, Brackett BG. Effects of developmental stage, embryonic interferon-tau secretion and recipient synchrony on pregnancy rate after transfer of in vitro produced bovine blastocysts. Reprod Domest Anim 2004; 39: 120-124

10. Heyman $\mathbf{Y}$, Chesne $\mathbf{P}$, Chupin D, Menezo Y. Improvement of survival rate of frozen cattle blastocysts after transfer with trophoblastic vesicles. Theriogenology 1987; 27 477-484.

11. Numabe T, Oikawa T, Kikuchi T, Horiuchi T. Production efficiency of Japanese black calves by transfer of bovine embryos produced in vitro. Theriogenology 2000; 54 1409-1420. 